\title{
MICROPROPAGATION AND ANTIOXIDANT ACTIVITY OF TARAXACUM FORMOSANUM KITAMURA - AN ENDANGERED MEDICINAL PLANT
}

\section{(c) Chang Hung-Chi ${ }^{1,3}$, Chen Jial-Jhen ${ }^{2}$, Karuppaiya Palaniyandi ${ }^{3}$, Tsay Hsin-Sheng ${ }^{3,4}$}

${ }^{1}$ Department of Golden-Ager Industry Management, Chaoyang University of Technology, Taichung, Taiwan ${ }^{2}$ School of Chinese Pharmaceutical Sciences and Chinese Medicine Resources, China Medical University, Taichung, Taiwan ${ }^{3}$ Institute of Biochemical Sciences and Technology, Chaoyang University of Technology, Taichung, Taiwan

${ }^{4}$ Department of Agronomy, National Chung Hsing University, Taichung, Taiwan

Taraxacum formosanum Kitamura (Taiwan dandelion) belongs to the family compositae and its whole plant extract commonly known as herbal tea is a popular folk drink and it has number of functional ingredients. The present study aims to develop a protocol for in vitro micropropagation and its antioxidant activity assay. Maximum numbers of shoots were obtained from shoot tip cultured on half strength Murashige \& Skoog (1962) (MS) medium supplemented with $0.3 \mathrm{mg} / \mathrm{L}$ benzyladenine. The well-developed shoots were rooted on $1 / 4$ MS medium supplemented with $0.3 \mathrm{mg} / \mathrm{L}$ naphthaleneacetic acid. The rooted plantlets were successfully acclimatized in greenhouse with $100 \%$ survival rate. Antioxidant activity assay was evaluated in leaf and roots of $T$. formosanum. Resulting, water extract of TL (leaf collected from tissue culture plant which was transferred to the field and grown for 3 months) showed higher scavenging effect at the concentration of $0.25 \mathrm{mg} / \mathrm{mL}$ and higher ferric reducing antioxidant potential. On the other hand, water extract of leaf obtained from both TL and WL (leaf collected from nature) showed higher Trolox equivalent antioxidant capacity. Phytochemical analysis showed that higher total phenol content was observed in water extract of leaf from both TL and WL.

\section{PHYTOCHEMICAL ANALYSIS USING DIRECT ANALYSIS IN REAL TIME HIGH RESOLUTION MASS SPECTROMETRY}

\author{
(C) Chernetsova E. S. ${ }^{1,2}$, Crawford E. A. ${ }^{3}$, Shikov A. N. ${ }^{4}$, \\ Pozharitskaya O. N. ${ }^{4}$, Makarov V. G. ${ }^{4}$, Morlock G. E. ${ }^{1,5}$
}

\author{
${ }^{1}$ Institute of Food Chemistry, University of Hohenheim, Garbenstrasse 28, 70599 Stuttgart, Germany \\ 2On leave from Research and Innovation Department, Peoples' Friendship University of Russia, Miklukho-Maklaya st. 6, \\ 117198 Moscow, Russia; chern_es@mail.ru \\ ${ }^{3}$ IonSense Inc., 999 Broadway, Suite 404, Saugus, MA 01906, USA \\ ${ }^{4}$ St-Petersburg Institute of Pharmacy, 47/5, Piskarevsky prospect, 195067, St-Petersburg, Russia \\ ${ }^{5}$ Institute of Nutritional Science, Justus-Liebig-University of Giessen, Heinrich-Buff-Ring 26, 35392 Giessen, Germany
}

The first publication on Direct Analysis in Real Time (DART) mass spectrometry (MS) appeared in 2005 (1), and since then DART-MS is rapidly emerging. Its main advantage is the minimization or even absence of sample preparation. In this study, the capabilities of a new ID-CUBE DART ion source for phytochemical analysis are investigated for the first time, interfacing it to Orbitrap MS and analyzing the extracts of the leaves of medicinal plants (Bergenia crassifolia) and of propolis. The simple sample preparation of extracts includes the application of the sample onto the metal grid of the OpenSpot ${ }^{\mathrm{TM}}$ Card and the manual introduction of the card into the ion source. The elemental compositions were assigned to the abundant signals in the extract mass spectra. This demonstrates the high potential of this new MS technique in phytochemical analysis, especially in the identification of unknown components by their exact mass values. The major phenolic components of the investigated extracts were confirmed by their $[\mathrm{M}-\mathrm{H}]^{-}$ions. All benefits of high resolution MS are used upon data processing using the coupling of DART with the Orbitrap mass spectrometer. Thus, the effective combination of DART with Orbitrap mass spectrometer has been proven as a powerful small molecule identification tool for phytochemical analysis.

Reference: R. B. Cody, J. A. Laramee, H. Dupont Durst, Anal. Chem. 2005, 77, 2297.

Acknowledgments: This work was financially supported within the program Erasmus Mundus Action 2 "IAMONET-RU". 